\title{
Effect of irrigation intervals and soil types on yield and yield components of tomato plants artificially inoculated with Clavibacter Michiganensis subsp. Michiganensis
}

\author{
Ayesha Bibi* and Musharaf Ahmad \\ Department of Plant Pathology, University Of Agriculture Peshawar, Pakistan \\ *Corresponding author's email: ayeshabibi3@yahoo.com
}

Citation

Ayesha Bibi* and Musharaf Ahmad. Effect of irrigation intervals and soil types on yield and yield components of tomato plants artificially inoculated with Clavibacter Michiganensis subsp. Michiganensis. Pure and Applied Biology. Vol. 5, Issue 4, pp793-803. http://dx.doi.org/10.19045/bspab.2016.50100

\begin{tabular}{llll}
\hline Received: 12/04/2016 & Revised: 25/07/2016 & Accepted: 30/07/2016 & Online First: 08/08/2016 \\
\hline \hline
\end{tabular}

\section{Abstract}

To determine the effect of irrigation interval and soil type on the severity of bacterial canker of tomato, a field experiment was carried out at Agricultural Research Institute (ARI) Tarnab in March to June 2013 and 2014. Five treatments: T1 = irrigation with 8 days interval, T2 = irrigation with 9 days interval, T3 = irrigation with 10 days interval, T4 = irrigation with 11 days interval and T5 = irrigation with 12 days interval were investigated for their effect on bacterial canker of tomato. In the same experiment, another factor i.e. soil types $(\mathrm{S} 1=$ Sandy Loam, S2 = Loam and S3 = Silt loam) were also investigated. Trials were replicated four times in a completely randomized design with split plot arrangement. Data were recorded for; survival percentage, number of flowers plant ${ }^{-1}$, number of fruits plant ${ }^{-1}$, fruiting percentage, disease severity and yield plant $^{-1}$. Results revealed that keeping irrigation interval at 12 days, the disease rating score reduced to its minimum (0.83), however the yield (1.88 kg.plant $\left.{ }^{-1}\right)$ was affected due to drought. For soil types less (1.67 and 1.77) disease severity was scored for sandy loam and silt loam soil respectively, whereas for the loam soil higher (2.10) disease ratings were observed. Highest $(2.29 \mathrm{~kg})$ yield per plant was recorded for silt loam, followed by loam soil with $2.17 \mathrm{~kg}$.plant ${ }^{-1}$. While 10 days irrigation interval was found to be the best with $2.48 \mathrm{~kg}$ yield per plant. It was therefore, concluded, that ten days interval in irrigation was found to be optimum for obtaining the maximum yield, while silt loam soil is best due to its optimum water-holding capacities for tomato crop to tolerate the bacterial canker disease.

Keywords: Irrigation intervals; Soil types; Bacterial canker; Tomato; Cmm.

Introduction

Tomato (Solanum lycopersicum), a native of South America is grown worldwide for its edible fruits, with thousands of cultivars having been selected with varying fruit types, and for optimum growth in differing growing conditions. Tomato consumption is believed to benefit the heart, reducing cardiovascular 
risk associated with type 2 diabetes [1]. Tomato contain the carotene lycopene, one of the most powerful natural antioxidants. In some studies, lycopene, especially in cooked tomatoes, has been found to help prevent prostate cancer. Lycopene has also been shown to improve the skin's ability to protect against harmful UV rays. Moreover, tomato consumption has been associated with decreased risk of breast cancer [2], head and neck cancers [3] and it is strongly protective against neurodegenerative diseases $[4,6]$.

In the year 2010, about 145.75 million tonnes of tomatoes were produced globally. Pakistan ranks $40^{\text {th }}$ in tomato production with a total production of 476826 tonnes during 2010[7]. In year 2011-12 the annual production of tomatoes in Pakistan was estimated around 530,000 tonnes while total area under the crop was 52300 hectares. For the same years in Khyber Pakhtunkhwa (KP) province total area under tomato crop was 12600 hectares with total production of 113200 tonnes [8]. Rise of the fast food industry in the country is also having a significant impact on the demand for tomato based products. It is expected that this trend will continue in the near future and the consumption of tomato will increase further.

The average per hectare production of tomato in KP is well below its potential. One of the reasons for this low yield and quality is the occurrence of different diseases. Tomato diseases are caused by Viruses, Nematodes, Fungi and Bacteria. Mildews, blights, wilts and cankers are some of the common diseases. Tomato canker, also known as Bacterial canker or bird's eye, is one of the most important bacterial diseases. The disease is caused by bacterium Clavibacter michiganensis subsp. michiganensis $(\mathrm{Cmm})$. Experiments on bacterial canker carried out in France have shown a yield loss of $20-30 \%$ [9], however in greenhouses it is reported that disease incidence reached from $10 \%$ to even $80 \%$ [10].
Tomato is not resistant to drought. Yields decrease considerably after short periods of water deficiency. The amount of water that is needed depends on the type of soil and on the weather conditions (amount of rain, humidity and temperature). About $20 \mathrm{~mm}$ of water per week is needed under cool conditions, about $70 \mathrm{~mm}$ during hot and dry periods. Irrigation with an interval of 7-8 days is recommended. The irrigation interval can be decreased from 5-6 days when weather is too hot [11].

The disease is very difficult to control. However, different control measures could be integrated to keep the disease under check. Since not a lot of scientific work has been done on plant bacterial diseases in KP province of Pakistan including bacterial canker, it is difficult to adopt the control measures that might bring the disease below thresh-hold level. Keeping in view the importance of the disease, it's very destructive nature and the high probability of its presence in KP, the present research study was initiated with the objective to manage the disease through irrigation intervals and soil types which are economical and effective components of the integrated disease management (IDM) against the disease.

\section{Materials and methods \\ Experimental layout}

In order to determine the effect of irrigation interval and soil type on the severity of bacterial canker of tomato, a field experiment was carried out at Agricultural Research Institute Tarnab, Peshawar in March to June 2013 and 2014. Because tomato is irrigated weekly for the first month after transplanting, and then every 10 days until crop completion [12], therefore, following five treatments: T1 $=$ irrigation with 8 days interval, $\mathrm{T} 2=$ irrigation with 9 days interval, T3 = irrigation with 10 days interval, T4 = irrigation with 11 days interval, T5 = irrigation with 12 days interval were investigated for their effect on bacterial canker of tomato. In the same experiment, another factor i.e. soil types (i.e. 
S1 = Sandy Loam, S2 = Loam and S3 = Silt loam) was also investigated as soil types play role in retaining soil water for considerable duration of time. For this purpose the three types of soil was brought from nearby districts i.e. Charsadda and Nowshera (as per soil texture data provided by Soil Chemistry Department, Agricultural Research Institute, Tarnab, Peshawar) for experimental seed beds. Trials were replicated four times in a completely randomized design with factorial arrangement. In each experimental units five plants were left un-inoculated as control for comparison, however, due to the nature of experiment and the statistical design used for analysis, it was excluded while analyzing the data.

\section{Nursery raising and field preparation}

Tomato nursery (Reo-Grand variety) was raised in trays. Before seedlings transplantation, the experimental plots were ploughed well and mixed with well-rotten farm yard manure. The beds were raised 12 $\mathrm{cm}$ above the soil surface to provide drainage for the surplus irrigation or excess rain water. Seedlings were transplanted at the spacing of $1.5 \mathrm{~m}$ between rows and $45 \mathrm{~cm}$ between plants. Standard cultural practices were adopted for all the treatments. Urea and DAP (di-ammonium-phosphate) were applied at standard and recommended rates for nitrogen and phosphorus requirements of the crop.

\section{Bacterial inoculation}

Bacterial isolate Cmm1-MKD1 (from the culture bank of plant pathology laboratory, The University of agriculture Peshawar) was used in the study. Bacterial culture was grown on commercially available Nutrient Agar (NA) Medium. The 72 hours old growing colonies were suspended in sterile saline $(0.85 \%)$ to adjust its concentration $10^{9}$ $\mathrm{cfu} / \mathrm{ml}\left(\mathrm{OD}_{600}\right)$ with spectrophotometer. In order to provide $\mathrm{Cmm}$ inoculum, the roots of the transplants were dipped in bacterial suspension just before transplantation.

\section{Data parameters}

Data were recorded on different parameters i.e. survival percentage, number of flowers per plant, number of fruits per plant, fruiting percentage (percent flowers resulted to fruits), disease severity and yield per plant. Evaluation of disease appearance and development was determined by using the scale of Foster and Echandi [13] with some modifications (index point 5 was added to the previous scale) (Table 1).

Table 1. Modified Disease severity scale, Foster and Echandi (1973)

\begin{tabular}{|c|c|l|}
\hline S. No & Ratings & Description \\
\hline 1 & 0 & No symptoms \\
\hline 2 & 1 & Up to $1 / 3$ of the leaves wilted \\
\hline 3 & 2 & Up to $2 / 3$ of the leaves wilted \\
\hline 4 & 3 & $\begin{array}{l}\text { More than } 2 / 3 \text { of wilted leaves. terminal leaves on the main shoot } \\
\text { not wilted }\end{array}$ \\
\hline 5 & 4 & Terminal leaves of the main shoot and most leaves wilted or dead \\
\hline 6 & 5 & Plant completely dead \\
\hline
\end{tabular}

\section{Results}

\section{Survival percentage}

Data revealed that survival of the plants is adversely affected by the pathogen when inoculated at the time of transplantation (Table 2). Significant differences among the soil types and irrigation intervals were recorded for both the years (Table 8). Loam soil type proved to be the best in terms of plant survival where $78.60 \%$ plants survived. It was followed by silt loam with $73.23 \%$ plant survival, while in sandy loam less $(70.87 \%)$ number of plants survived.

For irrigation intervals maximum (78.67 and $77.11 \%$ ) numbers of plants survived when irrigated at 10 and 11 days intervals 
respectively. However, minimum $(67.94 \%)$ number of plant survival recorded for 8 days irrigation interval.

Table 2. Effect of irrigation intervals and soil types on survival percentage of tomato plants inoculated with Cmm (Spring 2013, 2014)

\begin{tabular}{|c|c|c|c|c|c|c|c|}
\hline \multirow[t]{2}{*}{ Year } & \multirow[t]{2}{*}{ Soil types } & \multicolumn{5}{|c|}{ Irrigation intervals } & \multirow[t]{2}{*}{ Y $\times$ ST } \\
\hline & & 8 days & 9 days & 10 days & 11 days & 12 days & \\
\hline \multirow[t]{3}{*}{2013} & Sandy Loam & 60.67 & 73.00 & 72.67 & 70.33 & 69.33 & 69.20 \\
\hline & Loam & 72.00 & 81.00 & 89.67 & 75.67 & 78.00 & 79.27 \\
\hline & Silt loam & 69.33 & 70.67 & 74.67 & 84.67 & 71.00 & 74.07 \\
\hline \multirow[t]{4}{*}{2014} & Sandy Loam & 65.33 & 73.00 & 77.33 & 74.33 & 72.67 & 72.53 \\
\hline & Loam & 72.67 & 78.33 & 84.00 & 76.67 & 78.00 & 77.93 \\
\hline & Silt loam & 67.67 & 70.67 & 73.67 & 81.00 & 69.00 & 72.40 \\
\hline & & \multicolumn{5}{|c|}{ Year x Irrigation intervals } & Means \\
\hline 2013 & & 67.33 & 74.89 & 79.00 & 76.89 & 72.78 & 74.18 \\
\hline \multirow[t]{5}{*}{2014} & & 68.56 & 74.00 & 78.33 & 77.33 & 73.22 & 74.29 \\
\hline & & \multicolumn{5}{|c|}{ Soil types $\mathrm{x}$ Irrigation intervals } & Means \\
\hline & Sandy Loam & $\begin{array}{c}63.00 \\
\text { I }\end{array}$ & $\begin{array}{c}73.00 \\
\text { FG }\end{array}$ & $\begin{array}{l}75.00 \\
\text { DEF }\end{array}$ & $\begin{array}{c}72.33 \\
\text { FG }\end{array}$ & $\begin{array}{c}71.00 \\
\mathrm{GH}\end{array}$ & $\begin{array}{c}70.87 \\
\text { C }\end{array}$ \\
\hline & Loam & $\begin{array}{c}72.33 \\
\text { FG }\end{array}$ & $\begin{array}{c}79.67 \\
\text { C }\end{array}$ & $\begin{array}{c}86.83 \\
\text { A }\end{array}$ & $\begin{array}{c}76.17 \\
\mathrm{DE}\end{array}$ & $\begin{array}{c}78.00 \\
\text { CD }\end{array}$ & $\begin{array}{c}78.60 \\
\mathrm{~A}\end{array}$ \\
\hline & Silt loam & $\begin{array}{c}68.50 \\
\mathrm{H}\end{array}$ & $\begin{array}{c}70.67 \\
\mathrm{GH}\end{array}$ & $\begin{array}{c}74.17 \\
\mathrm{EF}\end{array}$ & $\begin{array}{c}82.83 \\
\text { B }\end{array}$ & $\begin{array}{c}70.00 \\
\mathrm{GH}\end{array}$ & $\begin{array}{c}73.23 \\
\text { B }\end{array}$ \\
\hline & Means & $\begin{array}{c}67.94 \\
C\end{array}$ & $\begin{array}{c}74.44 \\
\text { B }\end{array}$ & $\begin{array}{c}78.67 \\
\text { A }\end{array}$ & $\begin{array}{c}77.11 \\
\text { A }\end{array}$ & $\begin{array}{c}73.00 \\
\text { B }\end{array}$ & \\
\hline
\end{tabular}

Means followed by different letters in categories are significantly different at $5 \%$ level of probability LSD $(\mathrm{P} \leq 0.05)$ for Years $=1.12$, LSD $(\mathrm{P} \leq 0.05)$ for Soil types $=1.37$, LSD $(\mathrm{P} \leq 0.05)$ for Irrigation intervals $=1.76$, LSD $(\mathrm{P} \leq 0.05)$ for Years $x$ Soil types $=1.93$, LSD $(\mathrm{P} \leq 0.05)$ for Years $\mathrm{x}$ Irrigation intervals $=2.50$, LSD $(\mathrm{P} \leq 0.05)$ for Soil types $\mathrm{x}$ Irrigation intervals $=3.06$, LSD $(\mathrm{P} \leq 0.05)$ for Years $\mathrm{x}$ Soil types $\mathrm{x}$ Irrigation intervals $=4.32$

\section{Number of flowers plant ${ }^{-1}$}

Number of flowers (Table 3) produced on inoculated plants increased when irrigation interval increased from 8 to 10 days but this trend was reversed when irrigation interval was increased further up to 12 days. Maximum number of flowers (71.67) were produced when irrigation interval was 10 days, followed by 63.78 and 61.67 average number of flowers, produced on plants irrigated with 11 and 9 days intervals. Minimum (56.39 and 56.11) number of flowers were recorded when irrigation intervals were 8 and 12 days. On the other hand plants grown on Loamy soil produced maximum (66.07) numbers of flowers, while plants grown on sandy and silt loamy soil produced significantly less (59.20 and 60.50) number of flowers. 
Table 3.Effect of irrigation intervals and soil types on number of flowers $\left(\right.$ plant $\left.^{-1}\right)$ of tomato plants inoculated with Cmm (Spring 2013, 2014)

\begin{tabular}{|c|c|c|c|c|c|c|c|}
\hline \multirow[t]{2}{*}{ Year } & \multirow[t]{2}{*}{ Soil types } & \multicolumn{5}{|c|}{ Irrigation intervals } & \multirow[t]{2}{*}{ Y X ST } \\
\hline & & 8 days & 9 days & 10 days & 11 days & 12 days & \\
\hline \multirow[t]{3}{*}{2013} & Sandy Loam & 51.67 & 56.33 & 65.33 & 58.67 & 53.00 & 57.00 \\
\hline & Loam & 59.33 & 63.00 & 70.33 & 66.67 & 56.00 & 63.07 \\
\hline & Silt loam & 53.67 & 58.67 & 69.33 & 56.00 & 52.33 & 58.00 \\
\hline \multirow[t]{4}{*}{2014} & Sandy Loam & 53.67 & 60.33 & 71.33 & 64.67 & 57.00 & 61.40 \\
\hline & Loam & 63.33 & 69.00 & 77.33 & 73.67 & 62.00 & 69.07 \\
\hline & Silt loam & 56.67 & 62.67 & 76.33 & 63.00 & 56.33 & 63.00 \\
\hline & & \multicolumn{5}{|c|}{ Year x Irrigation intervals } & Means \\
\hline 2013 & & 54.89 & 59.33 & 68.33 & 60.44 & 53.78 & 59.36 \\
\hline \multirow[t]{5}{*}{2014} & & 57.89 & 64.00 & 75.00 & 67.11 & 58.44 & 64.49 \\
\hline & & \multicolumn{5}{|c|}{ Soil types x Irrigation intervals } & Means \\
\hline & Sandy Loam & $\begin{array}{c}52.67 \\
F\end{array}$ & $\begin{array}{c}58.33 \\
\mathrm{DE}\end{array}$ & $\begin{array}{c}68.33 \\
\text { BC }\end{array}$ & $\begin{array}{c}61.67 \\
\text { D }\end{array}$ & $\begin{array}{c}55.00 \\
\mathrm{EF}\end{array}$ & $\begin{array}{c}59.20 \\
\text { B }\end{array}$ \\
\hline & Loam & $\begin{array}{c}61.33 \\
D\end{array}$ & $\begin{array}{c}66.00 \\
C\end{array}$ & $\begin{array}{c}73.83 \\
\mathrm{~A}\end{array}$ & $\begin{array}{c}70.17 \\
\mathrm{AB}\end{array}$ & $\begin{array}{c}59.00 \\
\text { D }\end{array}$ & $\begin{array}{c}66.07 \\
\text { A }\end{array}$ \\
\hline & Silt loam & $\begin{array}{c}55.17 \\
\mathrm{EF}\end{array}$ & $\begin{array}{c}60.67 \\
\text { D }\end{array}$ & $\begin{array}{c}72.83 \\
\mathrm{~A} \\
\end{array}$ & $\begin{array}{c}59.50 \\
\text { D } \\
\end{array}$ & $\begin{array}{c}54.33 \\
F\end{array}$ & $\begin{array}{c}60.50 \\
\text { B }\end{array}$ \\
\hline & Means & $\begin{array}{c}56.39 \\
\mathrm{C}\end{array}$ & $\begin{array}{c}61.67 \\
\text { B }\end{array}$ & $\begin{array}{c}71.67 \\
\text { A }\end{array}$ & $\begin{array}{c}63.78 \\
\text { B }\end{array}$ & $\begin{array}{c}56.11 \\
\mathrm{C}\end{array}$ & \\
\hline
\end{tabular}

Means followed by different letters in categories are significantly different at $5 \%$ level of probability

LSD $(\mathrm{P} \leq 0.05)$ for Years $=1.36$, LSD $(\mathrm{P} \leq 0.05)$ for Soil types $=1.67$, LSD $(\mathrm{P} \leq 0.05)$ for Irrigation intervals $=2.15$, LSD $(\mathrm{P} \leq 0.05)$ for Years $x$ Soil types $=2.36$, LSD $(\mathrm{P} \leq 0.05)$ for Years $\mathrm{x}$ Irrigation intervals $=3.04$, LSD $(\mathrm{P} \leq 0.05)$ for Soil types $x$ Irrigation intervals $=3.73, \mathrm{LSD}(\mathrm{P} \leq 0.05)$ for Years $\mathrm{x}$ Soil types $\mathrm{x}$ Irrigation intervals $=5.27$

\section{Fruiting percentage}

Data (Table 4) showed that fruiting percentages were highly significant for soil types and irrigation intervals. Increase in irrigation intervals significantly increased fruiting percentage and maximum $(82.99 \%)$ number of flowers resulted into fruits on plants irrigated at 12 days interval, followed by plants irrigated at 11 days interval with $79.37 \%$ fruiting. Less $(70.61 \%)$ number of flowers produced fruits when irrigation interval was 8 days. Interaction effects were significant for fruiting percentage and maximum $(84.06 \%)$ fruiting was recorded in plants grown in sandy loam soil with 12 days irrigation intervals. In main effects plants grown on sandy loam, Loam and silt loam soil produced minimum (68.48 to $72.31 \%$ ) numbers of fruits from flowers. 
Table 4. Effect of irrigation intervals and soil types on fruiting percentage of tomato plants inoculated with Cmm (Spring 2013, 2014)

\begin{tabular}{|c|c|c|c|c|c|c|c|}
\hline \multirow[t]{2}{*}{ Year } & \multirow[t]{2}{*}{ Soil types } & \multicolumn{5}{|c|}{ Irrigation intervals } & \multirow[t]{2}{*}{ Y x ST } \\
\hline & & 8 days & 9 days & 10 days & 11 days & 12 days & \\
\hline \multirow[t]{3}{*}{2013} & Sandy Loam & 72.04 & 76.14 & 79.01 & 83.17 & 85.87 & 79.25 \\
\hline & Loam & 69.17 & 69.91 & 74.85 & 77.49 & 81.62 & 74.61 \\
\hline & Silt loam & 70.89 & 75.18 & 76.21 & 80.49 & 80.78 & 76.71 \\
\hline \multirow[t]{4}{*}{2014} & Sandy Loam & 72.58 & 75.06 & 77.08 & 79.34 & 82.25 & 77.26 \\
\hline & Loam & 67.79 & 70.49 & 73.27 & 77.40 & 83.37 & 74.47 \\
\hline & Silt loam & 71.20 & 74.58 & 76.61 & 78.33 & 84.05 & 76.95 \\
\hline & & \multicolumn{5}{|c|}{ Year x Irrigation intervals } & Means \\
\hline 2013 & & 70.70 & 73.74 & 76.69 & 80.38 & 82.76 & 76.86 \\
\hline \multirow{7}{*}{2014} & & 70.52 & 73.38 & 75.66 & 78.36 & 83.22 & 76.23 \\
\hline & & \multicolumn{5}{|c|}{ Soil types x Irrigation intervals } & Means \\
\hline & Sandy Loam & $\begin{array}{c}72.31 \\
\text { F-I }\end{array}$ & $\begin{array}{c}75.60 \\
D-G\end{array}$ & $\begin{array}{c}78.05 \\
\text { B-E }\end{array}$ & $\begin{array}{l}81.25 \\
\mathrm{ABC}\end{array}$ & $\begin{array}{c}84.06 \\
\text { A }\end{array}$ & $\begin{array}{c}78.253 \\
\text { A }\end{array}$ \\
\hline & Loam & 68.48 & 70.20 & 74.06 & 77.44 & 82.50 & 74.537 \\
\hline & & I & $\mathrm{HI}$ & E-H & $\mathrm{B}-\mathrm{F}$ & $\mathrm{AB}$ & $\mathrm{B}$ \\
\hline & Silt loam & 71.05 & 74.88 & 76.41 & 79.41 & 82.42 & 76.833 \\
\hline & & GHI & $\mathrm{D}-\mathrm{H}$ & $\mathrm{C}-\mathrm{F}$ & A-D & $\mathrm{AB}$ & $\mathrm{AB}$ \\
\hline & Means & 70.61 & 73.56 & 76.17 & 79.37 & 82.99 & \\
\hline & & $\mathrm{D}$ & CD & $\mathrm{C}$ & $\mathrm{B}$ & A & \\
\hline
\end{tabular}

Means followed by different letters in categories are significantly different at $5 \%$ level of probability

LSD $(\mathrm{P} \leq 0.05)$ for Years $=1.91$, LSD $(\mathrm{P} \leq 0.05)$ for Soil types $=2.34$, LSD $(\mathrm{P} \leq 0.05)$ for Irrigation intervals $=3.02$, LSD $(P \leq 0.05)$ for Years $x$ Soil types $=3.30$, LSD $(P \leq 0.05)$ for Years $x$ Irrigation intervals $=4.26, \operatorname{LSD}(\mathrm{P} \leq 0.05)$ for Soil types $\mathrm{x}$ Irrigation intervals $=5.22, \mathrm{LSD}(\mathrm{P} \leq 0.05)$ for Years $\mathrm{x}$ Soil types $\mathrm{x}$ Irrigation intervals $=7.39$

\section{Number of fruits plant ${ }^{-1}$}

The trend of fruits produced on inoculated plants was similar to that of flowers (Table 5). Maximum Number of fruits produced on inoculated plants increased when irrigation interval increased from 8 to 10 days but further increase in irrigation interval (up to 12 days) significantly reduced number of fruits. Maximum number fruits (54.44) were produced when irrigation interval was 10 days, followed by 50.50 average numbers of fruits, produced on plants irrigated with 11 days intervals. Minimum (39.78) number of fruits were recorded when irrigation intervals was 8 days. On the other hand plants grown on Loam soil produced maximum (49.20) numbers of fruits, while plants grown on sandy and silt loamy soil produced significantly less (46.37 each) number of fruits. 
Table 5. Effect of irrigation intervals and soil types on number of fruits of tomato plants inoculated with Cmm (Spring 2013, 2014)

\begin{tabular}{|c|c|c|c|c|c|c|c|}
\hline \multirow[t]{2}{*}{ Year } & \multirow[t]{2}{*}{ Soil types } & \multicolumn{5}{|c|}{ Irrigation intervals } & \multirow[t]{2}{*}{ Y $\times$ ST } \\
\hline & & 8 days & 9 days & 10 days & 11 days & 12 days & \\
\hline \multirow[t]{3}{*}{2013} & Sandy Loam & 37.33 & 43.00 & 51.67 & 48.67 & 45.33 & 45.20 \\
\hline & Loam & 41.00 & 44.00 & 52.67 & 51.67 & 45.67 & 47.00 \\
\hline & Silt loam & 38.00 & 44.00 & 52.33 & 45.00 & 42.33 & 44.33 \\
\hline \multirow[t]{4}{*}{2014} & Sandy Loam & 39.00 & 45.33 & 55.00 & 51.33 & 47.00 & 47.53 \\
\hline & Loam & 43.00 & 48.67 & 56.67 & 57.00 & 51.67 & 51.40 \\
\hline & Silt loam & 40.33 & 46.67 & 58.33 & 49.33 & 47.33 & 48.40 \\
\hline & & \multicolumn{5}{|c|}{ Year x Irrigation intervals } & Means \\
\hline 2013 & & 38.78 & 43.67 & 52.22 & 48.44 & 44.44 & 45.51 \\
\hline \multirow[t]{5}{*}{2014} & & 40.78 & 46.89 & 56.67 & 52.56 & 48.67 & 49.11 \\
\hline & & \multicolumn{5}{|c|}{ Soil types $x$ Irrigation intervals } & Means \\
\hline & Sandy Loam & $\begin{array}{c}38.17 \\
\mathrm{H}\end{array}$ & $\begin{array}{c}44.17 \\
\mathrm{EF}\end{array}$ & $\begin{array}{c}53.33 \\
\mathrm{AB}\end{array}$ & $\begin{array}{c}50.00 \\
\mathrm{BC}\end{array}$ & $\begin{array}{c}46.17 \\
\mathrm{DE}\end{array}$ & $\begin{array}{c}46.37 \\
\text { B }\end{array}$ \\
\hline & Loam & $\begin{array}{c}42.00 \\
\text { FG }\end{array}$ & $\begin{array}{l}46.33 \\
\text { CDE }\end{array}$ & $\begin{array}{c}54.67 \\
\text { A }\end{array}$ & $\begin{array}{c}54.33 \\
\text { A }\end{array}$ & $\begin{array}{c}48.67 \\
\text { CD }\end{array}$ & $\begin{array}{c}49.20 \\
\text { A }\end{array}$ \\
\hline & Silt loam & $\begin{array}{c}39.17 \\
\mathrm{GH}\end{array}$ & $\begin{array}{c}45.33 \\
\text { DEF }\end{array}$ & $\begin{array}{c}55.33 \\
\text { A }\end{array}$ & $\begin{array}{l}47.17 \\
\text { CDE }\end{array}$ & $\begin{array}{c}44.83 \\
\text { EF }\end{array}$ & $\begin{array}{c}46.37 \\
\text { B }\end{array}$ \\
\hline & Means & $\begin{array}{c}39.78 \\
\text { D }\end{array}$ & $\begin{array}{c}45.28 \\
C\end{array}$ & $\begin{array}{c}54.44 \\
\text { A }\end{array}$ & $\begin{array}{c}50.50 \\
\text { B }\end{array}$ & $\begin{array}{c}46.56 \\
C\end{array}$ & \\
\hline
\end{tabular}

Means followed by different letters in categories are significantly different at $5 \%$ level of probability

LSD $(\mathrm{P} \leq 0.05)$ for Years $=1.36, \mathrm{LSD}(\mathrm{P} \leq 0.05)$ for Soil types $=1.66, \mathrm{LSD}(\mathrm{P} \leq 0.05)$ for Irrigation intervals $=2.15$, LSD $(\mathrm{P} \leq 0.05)$ for Years $x$ Soil types $=2.35$, LSD $(\mathrm{P} \leq 0.05)$ for Years $x$ Irrigation intervals $=3.03$, LSD $(\mathrm{P} \leq 0.05)$ for Soil types $\mathrm{x}$ Irrigation intervals $=3.72, \mathrm{LSD}(\mathrm{P} \leq 0.05)$ for Years $\mathrm{x}$ Soil types $\mathrm{x}$ Irrigation intervals $=5.25$

\section{Disease severity}

Data pertaining to disease severity of bacterial canker of tomato are shown in Table 6. Disease ratings were highly significant for soil types and irrigation intervals (Table 8). Highest disease rating (3.00) was recorded when plants irrigated on $8^{\text {th }}$ day, followed by 9 days irrigation interval and so on. However, when irrigation interval increased to 12 days, the disease reduced to its minimum (0.83) score. On the other hand less (1.67 and 1.77) disease severity was recorded for sandy loam and silt loam soil respectively, whereas for the loam soil higher (2.10) disease ratings were observed. In year wise comparison for both the years average disease ratings recorded were the same i.e. 1.84. 
Table 6. Effect of irrigation intervals and soil types on disease severity of tomato plants inoculated with Cmm (Spring 2013, 2014)

\begin{tabular}{|c|c|c|c|c|c|c|c|}
\hline \multirow[t]{2}{*}{ Year } & \multirow[t]{2}{*}{ Soil types } & \multicolumn{5}{|c|}{ Irrigation intervals } & \multirow[t]{2}{*}{ Y X ST } \\
\hline & & 8 days & 9 days & 10 days & 11 days & 12 days & \\
\hline \multirow{3}{*}{2013} & Sandy Loam & 3.00 & 2.67 & 1.33 & 0.33 & 0.00 & 1.47 \\
\hline & Loam & 3.33 & 2.33 & 2.33 & 2.00 & 1.00 & 2.20 \\
\hline & Silt loam & 3.33 & 2.67 & 1.33 & 1.00 & 1.00 & 1.87 \\
\hline \multirow[t]{4}{*}{2014} & Sandy Loam & 3.00 & 2.33 & 1.67 & 1.33 & 1.00 & 1.87 \\
\hline & Loam & 2.67 & 2.67 & 2.00 & 1.33 & 1.33 & 2.00 \\
\hline & Silt loam & 2.67 & 2.33 & 1.67 & 1.00 & 0.67 & 1.67 \\
\hline & & \multicolumn{5}{|c|}{ Year $\mathrm{x}$ Irrigation intervals } & Means \\
\hline 2013 & & 3.22 & 2.56 & 1.67 & 1.11 & 0.67 & 1.84 \\
\hline \multirow[t]{7}{*}{2014} & & 2.78 & 2.44 & 1.78 & 1.22 & 1.00 & 1.84 \\
\hline & & \multicolumn{5}{|c|}{ Soil types $\mathrm{x}$ Irrigation intervals } & Means \\
\hline & Sandy Loam & 3.00 & 2.50 & 1.50 & 0.83 & 0.50 & 1.67 \\
\hline & & A & $\mathrm{AB}$ & DE & FG & $\mathrm{G}$ & B \\
\hline & Loam & 3.00 & 2.50 & 2.17 & 1.67 & 1.17 & 2.10 \\
\hline & & A & $\mathrm{AB}$ & $\mathrm{BC}$ & $\mathrm{CD}$ & DEF & A \\
\hline & Silt loam & 3.00 & 2.50 & 1.50 & 1.00 & 0.83 & 1.77 \\
\hline & & A & $\mathrm{AB}$ & DE & EFG & FG & B \\
\hline & Means & 3.00 & 2.50 & 1.72 & 1.17 & 0.83 & \\
\hline & & A & B & $\mathrm{C}$ & $\mathrm{D}$ & $\mathrm{E}$ & \\
\hline
\end{tabular}

Means followed by different letters in categories are significantly different at 5\% level of probability. LSD (P $\leq 0.05)$ for Years $=0.193, \mathrm{LSD}(\mathrm{P} \leq 0.05)$ for Soil types $=0.237, \mathrm{LSD}(\mathrm{P} \leq 0.05)$ for Irrigation intervals $=0.306, \mathrm{LSD}(\mathrm{P} \leq 0.05)$ for Years $\mathrm{x}$ Soil types $=0.335$, LSD $(\mathrm{P} \leq 0.05)$ for Years $\mathrm{x}$ Irrigation intervals $=0.432$, LSD $(\mathrm{P} \leq 0.05)$ for Soil types $\mathrm{x}$ Irrigation intervals $=0.530, \operatorname{LSD}(\mathrm{P} \leq 0.05)$ for Years $\mathrm{x}$ Soil types $\mathrm{x}$ Irrigation intervals $=0.749$

\section{Yield (kg. plant ${ }^{-1}$ )}

Yield data for five pickings of inoculated tomato plants are shown in Table 7. Plant yield was highly significant for soil types and irrigation intervals (Table 8). Highest $(2.29 \mathrm{~kg})$ yield per plant was recorded for silt loam, followed by loam soil with $2.17 \mathrm{~kg}$.plant ${ }^{-1}$. The lowest $(2.03 \mathrm{~kg})$ yield per plant was recorded for sandy loam soil. Among irrigation intervals 10 days irrigation interval was proved to be the best with $2.48 \mathrm{~kg}$ yield per plant which was followed by both 9 and 11 days irrigation intervals with $2.23 \mathrm{~kg}$ and $2.21 \mathrm{~kg}$ yield per plant. The lowest (1.88kg.plant ${ }^{-1}$ ) yielding plants were those which irrigated at 12 days interval. For year 2014 all the varieties give comparatively less $(2.11 \mathrm{~kg})$ average yield as compared to that of $2.21 \mathrm{~kg}$ in 2013 .

\section{Discussion}

The basic factor that affects disease severity in most foliar pathosystems is leaf wetness [14], and among the agronomic practices are the irrigation type, dry season cropping and cultivation in protected environments [15]. Tomato is susceptible to drought and water deficiency even for a short periods, considerably reduce the yield. It is important to water plants regularly [16], especially during flowering and fruit formation. Water requirements of the crop strongly depends on the soil type and weather conditions (amount of rain, humidity and temperature). About 20 $\mathrm{mm}$ of water per week is needed under cool conditions, about $70 \mathrm{~mm}$ during hot and dry periods [11]. A considerable number of flowers were produced on the plants which successfully resulted in fruits too but still the average yield per plant was low because of 
smaller fruit size. It can be explained with the fact that watering plays a major role in attaining uniform maturity and reducing physiological disorders associated with

Table 7.Effect of irrigation intervals and soil types on yield (kg/plant) of tomato plants inoculated with Cmm (Spring 2013, 2014)

\begin{tabular}{|c|c|c|c|c|c|c|c|}
\hline \multirow[t]{2}{*}{ Year } & \multirow[t]{2}{*}{ Soil types } & \multicolumn{5}{|c|}{ Irrigation intervals } & \multirow[t]{2}{*}{ Y x ST } \\
\hline & & 8 days & 9 days & 10 days & 11 days & 12 days & \\
\hline \multirow[t]{3}{*}{2013} & Sandy Loam & 1.88 & 2.17 & 2.47 & 2.18 & 1.80 & 2.10 \\
\hline & Loam & 2.10 & 2.29 & 2.58 & 2.20 & 1.96 & 2.23 \\
\hline & Silt loam & 2.20 & 2.31 & 2.63 & 2.33 & 2.09 & 2.31 \\
\hline \multirow[t]{4}{*}{2014} & Sandy Loam & 1.78 & 2.13 & 2.21 & 2.12 & 1.58 & 1.96 \\
\hline & Loam & 1.97 & 2.18 & 2.43 & 2.16 & 1.82 & 2.11 \\
\hline & Silt loam & 2.15 & 2.33 & 2.55 & 2.25 & 2.02 & 2.26 \\
\hline & & \multicolumn{5}{|c|}{ Year x Irrigation intervals } & Means \\
\hline 2013 & & 2.06 & 2.25 & 2.56 & 2.24 & 1.95 & 2.21 \\
\hline \multirow[t]{6}{*}{2014} & & 1.96 & 2.21 & 2.40 & 2.18 & 1.81 & 2.11 \\
\hline & & \multicolumn{5}{|c|}{ Soil types $\mathrm{x}$ Irrigation intervals } & Means \\
\hline & Sandy Loam & $\begin{array}{c}1.83 \\
\mathrm{G}\end{array}$ & $\begin{array}{l}2.15 \\
\mathrm{DE}\end{array}$ & $\begin{array}{c}2.34 \\
\mathrm{~B}\end{array}$ & $\begin{array}{c}2.15 \\
\mathrm{DE}\end{array}$ & $\begin{array}{c}1.69 \\
\mathrm{H}\end{array}$ & $\begin{array}{c}2.03 \\
\mathrm{C}\end{array}$ \\
\hline & Loam & $\begin{array}{c}2.03 \\
\mathrm{~F}\end{array}$ & $\begin{array}{l}2.23 \\
\mathrm{CD}\end{array}$ & $\begin{array}{c}2.50 \\
\mathrm{~A}\end{array}$ & $\begin{array}{c}2.18 \\
\mathrm{D}\end{array}$ & $\begin{array}{c}1.89 \\
\mathrm{G}\end{array}$ & $\begin{array}{c}2.17 \\
\mathrm{~B}\end{array}$ \\
\hline & Silt loam & 2.17 & 2.32 & 2.59 & 2.29 & 2.06 & 2.29 \\
\hline & & $\mathrm{D}$ & $\mathrm{BC}$ & A & $\mathrm{BC}$ & $\mathrm{EF}$ & A \\
\hline & Means & $\begin{array}{c}2.01 \\
\mathrm{C}\end{array}$ & $\begin{array}{c}2.23 \\
\mathrm{~B}\end{array}$ & $\begin{array}{c}2.48 \\
\mathrm{~A}\end{array}$ & $\begin{array}{c}2.21 \\
\mathrm{~B}\end{array}$ & $\begin{array}{c}1.88 \\
D\end{array}$ & \\
\hline
\end{tabular}

Means followed by different letters in categories are significantly different at $5 \%$ level of probability LSD $(P \leq 0.05)$ for Years $=0.036, \mathrm{LSD}(\mathrm{P} \leq 0.05)$ for Soil types $=0.044, \mathrm{LSD}(\mathrm{P} \leq 0.05)$ for Irrigation intervals $=0.057$, LSD $(P \leq 0.05)$ for Years $x$ Soil types $=0.063$, LSD $(P \leq 0.05)$ for Years $x$ Irrigation intervals $=0.0810, \operatorname{LSD}(P \leq 0.05)$ for Soil types $x$ Irrigation intervals $=0.099, \operatorname{LSD}(\mathrm{P} \leq 0.05)$ for Years $\mathrm{x}$ Soil types $\mathrm{x}$ Irrigation intervals $=0.140$

Table 8. Planned comparison for significant interactions and main effects

\begin{tabular}{|l|l|l|l|l|l|l|}
\hline Effects & $\begin{array}{l}\text { \% } \\
\text { Survival }\end{array}$ & $\begin{array}{l}\text { No. of } \\
\text { flowers }\end{array}$ & $\begin{array}{l}\text { \% } \\
\text { Fruiting }\end{array}$ & $\begin{array}{l}\text { No. of } \\
\text { Fruits }\end{array}$ & $\begin{array}{l}\text { Disease } \\
\text { ratings }\end{array}$ & Yield \\
\hline Year (Y) & Ns & $* *$ & $\mathrm{~ns}$ & $* *$ & Ns & $* *$ \\
\hline Soil Type (S) & $* *$ & $* *$ & $* *$ & $* *$ & $* *$ & $* *$ \\
\hline Irrigation Interval (I) & $* *$ & $* *$ & $* *$ & $* *$ & $* *$ & $* *$ \\
\hline $\mathrm{Y} \times \mathrm{S}$ & $* *$ & $\mathrm{Ns}$ & $\mathrm{ns}$ & $\mathrm{ns}$ & $*$ & $\mathrm{Ns}$ \\
\hline $\mathrm{Y} \times \mathrm{I}$ & $\mathrm{Ns}$ & $\mathrm{Ns}$ & $\mathrm{ns}$ & $\mathrm{ns}$ & $\mathrm{Ns}$ & $\mathrm{Ns}$ \\
\hline $\mathrm{S} \times \mathrm{I}$ & $* *$ & $*$ & $\mathrm{~ns}$ & $\mathrm{~ns}$ & $\mathrm{Ns}$ & $*$ \\
\hline $\mathrm{Y} \times \mathrm{S} \times \mathrm{I}$ & $\mathrm{Ns}$ & $\mathrm{Ns}$ & $\mathrm{ns}$ & $\mathrm{ns}$ & $\mathrm{Ns}$ & $\mathrm{Ns}$ \\
\hline
\end{tabular}

$*=$ significant at $\mathrm{P} \leq 0.05, * *=$ significant at $\mathrm{P} \leq 0.01$, ns $=$ non-significant

Current research point out that soil moisture is directly proportional to disease i.e. more irrigations, keeps more soil moisture and thus results in more disease. Previous findings shows that 7-8 days irrigation interval is recommended but it can be decreased from 56 days when weather is too hot [11]. This statement is supported by the current 
investigations with the addition that availability of soil water is equally important to weather hotness. The amount of moisture present in soil at a particular time mostly depends on soil type. Loams and clay-loam soils have greater water-holding capacity than sandy-loam soils [11]

Though disease continuously reduced by increasing the irrigation interval, similarly an increase in yield was observed upto ten days interval, however, when the interval was further increased, drought adversely affected the yield which supports previous findings $[11,15,17]$.

In order to use all the possible control measures against the disease to keep it under control soil plays an important role in the early establishment of the disease. As the pathogen is soil-borne and have a close relation with the soil type and soil moisture therefore, experiments on soil moisture and soil types give some good information for managing the disease [17].

The amount of moisture present in soil at a particular time mostly depends on soil type. Plant wilting can be caused either by the plant or by the soil $[18,20]$ Soils having more water-holding capacity are more favorable for bacterial development and increase in soil moisture extended the survival of the endophytic bacteria [18]. Consistent with this notion, less disease severity was recorded for sandy loam soil as water holding capacity of sandy loam soil is less that silt loam and loam soil.

\section{Conclusion and recommendations}

It was concluded from the above results that, ten days interval in irrigation was proved to be optimum for obtaining the maximum yield, and silt loam soil was best for tomato crop to give good yield while keeping the disease under the threshold level. Therefore, it is recommended to the tomato growers to irrigate tomato crop after ten days regularly, while silt loam soil is the best soil to be used specially for green houses and tunnel farming.

Authors' contributions

Conceived and designed the experiments: A Bibi \& $M$ Ahmad. Performed the experiments: A Bibi. Analyzed the data: A Bibi. Contributed materials/analysis tools: A Bibi. Wrote the paper: A Bibi.

\section{Acknowledgments}

Higher Education Commission of Pakistan contributed as the only funding agency for this research.

\section{References}

1. Shidfar F, Froghifar N, Vafa M, Rajab A, Hosseini S, Shidfar S \& Gohari M (2011). The effects of tomato consumption on serum glucose, apolipoprotein B, apolipoprotein A-I, homocysteine and blood pressure in type 2 diabetic patients. International J Food Science Nutrition 62(3): 289-294.

2. Zhang CX, Ho SC, Chen YM, Fu JH, Cheng SZ \& Lin FY (2009). Greater vegetable and fruit intake is associated with a lower risk of breast cancer among Chinese women. International J Cancer 125(1): 181-188.

3. Freedman ND, Park Y, Subar AF, Hollenbeck AR, Leitzmann MF, Schatzkin A \& Abnet CC (2008). Fruit and vegetable intake and head and neck cancer risk in a large United States prospective cohort study. International J Cancer 122 (10): 2330-2336.

4. Rao AV \& Balachandran B (2002). Role of oxidative stress and antioxidants in neurodegenerative diseases. Nutr Neurosc 5(5): 291-309.

5. Fall PA, Fredrikson M, Axelson O \& Granérus AK (1999). Nutritional and occupational factors influencing the risk of Parkinson's disease: a case-control study in southeastern Sweden. Move Dis. 14 (1): 28-37.

6. Suganuma $\mathrm{H}$, Hirano $\mathrm{T}$, Arimoto $\mathrm{Y} \&$ Inakuma $\mathrm{T}$ (2002). Effect of tomato 
intake on striatal monoamine level in a mouse model of experimental Parkinson's disease. J Nutr Sci Vitm 48 (3): 251-254.

7. FAOSTAT (2010). FAO (Food and Agricultural Organization of the United Nations). http://faostat.fao.org.

8. FAOSTAT (2012). FAO (Food and Agricultural Organization of the United Nations). http://faostat.fao.org.

9. Rat B, Poissonnier J, Goisque MJ \& Burgaud A (1991). Le point sur le chancre bactérien. Fruit Légu. 86:38-40.

10. Vasinauskienë M (2002). Bacterial diseases of greenhouse-grown tomatoes. Biologiya (Viln.) 1: 29-31.

11. PAR (2015) Pakistan agriculture research, Agriculture education: tomato crop in Pakistan, http://edu.par.com.pk/wiki/tomato/

12. Hanson P, Chen JT, Kuo CG, Morris R \& Opena RT (2001) Tutorial on Tomato Production, irrigation. AVRDC, The World Vegetable Center.

13. Foster RL \& Echandi E (1973). Relation of age of plants, temperature and inoculum concentration to bacterial canker development in resistance and susceptible Lycopersicon spp. Phytopathol 63: 773-777.

14. Rotem J\& Palti J (1969). Irrigation and plant diseases. Annu Rev Phytopathol 7:267-288.
15. Diver S, Kuepper G \& Born H (1999). Organic tomato production [Online]. National Center for Appropriate Technology (ATTRA) Publication \#CT073/149. Available at: http://attra.ncat.org/attrapub/tomato.ht ml\#training (Last updated on: December 14, 2015).

16. Kakar KH, Amanullah \& Asif I (2015). Effect of irrigation levels and bedsystem of planting on seed fill duration, seed growth rate, yield and yield components of spring wheat (Triticum aestivum) under semiarid condition. Pure Appl Biol 4(4): 511-521.

17. Marshall KC (1975). Clay Mineralogy in Relation to Survival of Soil Bacteria. Annu Rev Phytopathol. 13: 357-373.

18. Oliveira ALM, Canuto EL, Silva EE, Reis VM \& Baldani JI (2004). Survival of endophytic diazotrophic bacteria in soil under different moisture levels. Brazil J Microb. 35:295-299.

19. Czyż EA \& Dexter AR (2012). Plant wilting can be caused either by the plant or by the soil. Soil Res. 50:708-713.

20. Czyż EA \& Dexter AR (2013). Influence of soil type on the wilting of plants. Inter. Agrophysics 27(4):385390. 